\title{
Olfactory receptor $51 E 1$ as a novel target for diagnosis in somatostatin receptor-negative lung carcinoids
}

\author{
Valeria Giandomenico, $^{1, *}$, Tao Cui ${ }^{2, *}$, Lars Grimelius ${ }^{3}$, Kjell Öberg ${ }^{1,2,4}$, \\ Giuseppe Pelosi ${ }^{5,6}$ and Apostolos V Tsolakis ${ }^{2}$ \\ 'Department of Medical Sciences, Endocrine Oncology, Science for Life Laboratory, Uppsala University Hospital, \\ Uppsala University, Entrance 70, 3rd Floor, Research Department 2, SE-751 85 Uppsala, Sweden \\ ${ }^{2}$ Department of Medical Sciences, Endocrine Oncology ${ }^{3}$ Department of Immunology, Genetics and Pathology, \\ Uppsala University, Uppsala, Sweden \\ ${ }^{4}$ Centre of Excellence for Endocrine Tumors, Uppsala University Hospital, Uppsala, Sweden \\ ${ }^{5}$ Division of Pathology and Laboratory Medicine, European Institute of Oncology, Milan, Italy \\ ${ }^{6}$ Department of Biomedical and Clinical Sciences 'Luigi Sacco', University of Milan School of Medicine, Milan, Italy \\ *(V Giandomenico and T Cui contributed equally to this work)
}

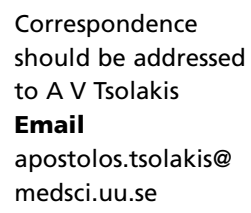

\begin{abstract}
Somatostatin receptors (SSTRs) may be used in lung carcinoids (LCs) for diagnosis and therapy, although additional targets are clearly warranted. This study aimed to investigate whether olfactory receptor $51 \mathrm{E} 1$ (OR51E1) may be a potential target for LCs. OR51E1 coding sequence was analyzed in $\mathrm{LC}$ cell lines, $\mathrm{NCl}-\mathrm{H} 727$ and $\mathrm{NCl}-\mathrm{H} 720$. OR51E1 transcript expression was investigated in LC cell lines and frozen specimens by quantitative real-time PCR. OR51E1, SSTR2, SSTR3, and SSTR5 expression was evaluated by immunohistochemistry on paraffin-embedded sections of 73 typical carcinoids (TCs), 14 atypical carcinoids (ACs), and 11 regional/distant metastases and compared with OctreoScan data. Immunohistochemistry results were rendered semiquantitatively on a scale from 0 to 3, taking into account the cellular compartmentalization (membrane vs cytoplasm) and the percentage of tumor cells ( $<50$ vs $>50 \%$ ). Our results showed that WT OR51E1 transcript was expressed in both LC cell lines. OR51E1 mRNA was expressed in 9 out of 12 TCs and 7 out of 9 ACs $(P=N S)$. Immunohistochemically, OR51E1, SSTR2, SSTR3, and SSTR5 were detected in 85, 71, 25, and $39 \%$ of TCs and in $86,79,43$, and $36 \%$ of ACs respectively. OR51E1 immunohistochemical scores were higher or equal than those of SSTRs' in $79 \%$ of TCs and $86 \%$ of ACs. Furthermore, in the LC cases where all SSTR subtypes were lacking, membrane OR51E1 expression was detected in 10 out of 17 TCs and 1 out of 2 ACs. Moreover, higher OR51E1 immunohistochemical scores were detected in 5 out of 6 OctreoScan-negative LC lesions. Therefore, the high expression of OR51E1 in LCs makes it a potential novel diagnostic target in SSTR-negative tumors.
\end{abstract}

\section{Key Words}

- olfactory receptor $51 \mathrm{E} 1$

- lung carcinoids

- novel target for diagnosis

- somatostatin receptors

- Octreoscan

\section{Introduction}

Neuroendocrine neoplasms of the respiratory tract are divided into well-differentiated tumors, i.e. lung carcinoids (LCs), and poorly differentiated neuroendocrine carcinomas, i.e. large cell neuroendocrine carcinomas and small-cell lung carcinomas (SCLCs). In turn, LCs are subdivided into typical carcinoids (TCs) and atypical
Journal of Molecular

Endocrinology

(2013) 51, 277-286 
carcinoids (ACs), the former being low-grade malignant tumors with less than two mitoses per $2 \mathrm{~mm}^{2}$, absence of necrosis, and good prognosis ( $\approx 88 \%$, 5-year survival) and the latter being intermediate-grade malignant tumors with up to ten mitoses per $2 \mathrm{~mm}^{2}$ and/or presence of punctate necrosis and poorer prognosis ( $\approx 50 \%$, 5-year survival) (Beasley et al. 2004). Differences in metastatic potential to regional lymph nodes, liver, and bone account for major differences in behavior of TCs and ACs (Beasley et al. 2004), with radical surgery offering the best chance to survive these tumors (Filosso et al. 2002a).

Because susceptibility to chemotherapy agents of metastatic LCs may be reduced due to low response rates and serious side effects, multimodality strategies have been developed (Gustafsson et al. 2008). Indeed, somatostatin receptors (SSTRs) can be expressed in LCs (Righi et al. 2010) and may be used for both their diagnosis and therapy (Pelosi et al. 2006). In particular, SSTR scintigraphy using ${ }^{111}$ In-DTPA ${ }^{0}$-octreotide (OctreoScan) has been applied to diagnose LCs targeting mainly SSTR2, with an additional affinity to SSTR3 and SSTR5 (Papotti et al. 2001). Somatostatin analog (SSA) biotherapy has been shown mainly to control symptoms and improve long-term survival of LC patients, especially those with metastatic ACs (Filosso et al. 2002b). Moreover, peptide receptor radionuclide therapy with radiolabeled SSAs, such as $\left[{ }^{177} \mathrm{Lu}^{-D O T A}{ }^{0}, \mathrm{Tyr}^{3}\right]$-octreotate (van Essen et al. 2007a), represents an alternative and valuable treatment in neuroendocrine tumor (NET) patients (Kwekkeboom et al. 2011), to cause tumor response or stabilization (van Essen et al. 2007a). However, NET patients with negative or low-level uptake in SSTR scintigraphy are not optimal candidates for using SSAs as diagnostic or therapeutic tools (van Essen et al. 2007b). Therefore, the development of novel tumor targets in LCs is clinically warranted to improve diagnosis and therapy.

The olfactory receptor (OR) genes belong to the largest superfamily in the human genome (Olender et al. 2008). ORs comprise a subclass of G-protein-coupled receptors predominantly expressed in the olfactory sensory neurons where they play a pivotal role in the specific recognition of diverse stimuli (Ache \& Young 2005). One of these genes, termed OR51E1 (family 51, subfamily E, member 1), is poorly understood due to the orphan status of its physiological ligand. However, odorants such as 3-methyl-valeric acid, 4-methyl-valeric acid (Fujita et al. 2007), nonanoic acid, and butyl butyryl lactate (Saito et al. 2009) have shown high affinity to human OR51E1. OR51E1 mRNA expression has been detected in normal brain (Vanti et al. 2003) and prostate tissue (Weigle et al. 2004). Furthermore, cancer cells, such as prostate carcinoma cells (Weigle et al. 2004, Fuessel et al. 2006, Wang et al. 2006, Weng et al. 2006) and small intestine neuroendocrine carcinoma (SI-NEC) cells (Leja et al. 2009, Cui et al. 2013), have been shown to express high levels of OR51E1. The pathophysiological role of OR51E1 in tumorigenesis and tumor progression has not been elucidated. However, OR51E1 has been suggested as a novel diagnostic marker complementary to other traditional markers in prostate cancers (Wang et al. 2006) and can significantly distinguish tumors from normal tissue and benign hyperplasia in the prostate (Weng et al. 2006). Although OR51E1 does not associate with the aggressiveness of prostate cancer, lower OR51E1 expression correlates with earlier recurrence (Weng et al. 2006).

The aim of this study was to investigate the OR51E1 coding sequence, the relevant protein and mRNA expression in LCs, normal lung tissue, various types of lung cancer, and established LC cell lines. The type of subcellular immunohistochemical localization of OR51E1 in LCs was compared with that of SSTR2, SSTR3, and SSTR5. The SSTR2, SSTR3, and SSTR5 protein expression was also correlated with the in vivo OctreoScan images in LC patients to further consolidate the possible diagnostic value of the SSTR immunohistochemical expression. Our results indicated that OR51E1 is likely to represent a novel target for diagnosis in LCs, alternative to SSTRs.

\section{Subjects and methods}

\section{Cell culture}

LNCaP cells, from a prostate carcinoma, NCI-H727 cells, from a TC, and NCI-H720 cells, from an AC, were purchased from American Type Culture Collection (ATCC, University Boulevard, Manassas, VA, USA) and cultured as recommended by the catalog. KRJ-1 and CNDT2.5, both SI-NEC cells, were gifts from Prof. I M Modlin (Yale University, New Haven, CT, USA) and Prof. L M Ellis (Anderson Cancer Centre, Houston, TX, USA) respectively and were cultured as described previously (Modlin et al. 2006, van Buren et al. 2007). Cell culture media components comprised DMEM-F12, F12, RPMI1640, sodium pyruvate, MEM vitamin, HEPES, and NEAA, all purchased from Life Technologies; PEST and L-glutamine from the National Veterinary Institute (SVA, Uppsala, Sweden); and FBS from Sigma.

Published by Bioscientifica Ltd. 


\section{Patients and tumors}

Frozen specimens were obtained from 31 patients with lung neuroendocrine neoplasms, including 12 TCs (three males and nine females), nine ACs (three males and six females), and ten SCLCs (five men and five females). Moreover, five lung adenocarcinomas and five squamous cell carcinomas, matched for age and sex, were used as a lung tumor-related control group. The tumor cell population was on average about $80 \%$ in each specimen. In addition, normal surrounding lung tissue was available from eight of the aforementioned LC patients (four TCs and four ACs).

Furthermore, the study included surgical specimens from 86 primary tumors (72 TCs and 14 ACs), ten lymph node metastases from TCs, and one liver metastasis from an AC. In addition, another laser-treated primary TC was also included. The specimens derived from a total of 80 patients, including 66 patients with TCs, of which six had multiple tumors and 14 patients with ACs. The median age at operation was 52 years for TC patients ( 22 males and 44 females) and 51 years for AC patients (five males and nine females). The average primary tumor size was $2.0 \pm$ $2.5 \mathrm{~cm}$ (mean \pm 2 s.D.) in TCs and $2.6 \pm 2.9 \mathrm{~cm}$ in ACs. Two patients with TCs emerged under the spectrum of multiple endocrine neoplasia type 1 syndrome. Four patients had clinical syndromes attributed to inappropriate hormone secretion from the tumor, two with Cushing's syndrome due to ectopic adrenocorticotrophin secretion, one with typical, and one with AC syndrome. Four TC patients had been treated before operation, one received paraplatin and etoposide, two temozolomide, and one interferon $\alpha$. Of these, one patient concomitantly received SSA for control of the carcinoid syndrome.

In 14 cases, i.e. 12 TC patients and two AC patients, after injecting $175-220 \mathrm{MBq}{ }^{111}$ In-DTPA-octreotide, OctreoScan was performed preoperatively and 19 focal lesions comprising 14 primary tumors, four lymph node metastases, and one liver metastasis were graded according to the method of Kwekkeboom et al. (2005).

\section{Controls}

Additional specimens from normal pancreas obtained from a patient operated on for a non-functioning pancreatic NET and specimens from a patient operated on for a prostate carcinoma (Gleason grade 5), were used as positive controls for the SSTRs and OR51E1 respectively. The specificity of all reactions was checked by replacing the primary antibody with a non-related rabbit immunoglobulin (rabbit IgG, cat no. I-1000) purchased from
Vector Labs (Burlingame, CA, USA) at a comparable dilution. Positive and negative controls were used as appropriate. In occasional cases, i.e. four primary TCs, one lymph node metastasis deriving from a TC case, and one liver metastasis from an AC, neutralization test was conducted by a $24-\mathrm{h}$ incubation of the primary antibody with the relevant antigen, i.e. $100 \mu \mathrm{g}$ antigen $/ \mathrm{ml}$ diluted antibody solution, before application to the sections. The antigens for SSTRs were obtained from Gramsch Laboratories (Schwabhausen, Germany): SSTR2A (SS-801), SSTR3 (SS-851), and SSTR5 (SS-891) and from Epitomics, Inc. (Burlingame, CA, USA): SSTR2 (P-3582-1); the antigen for OR51E1 (P1854) was from LSBio (Seattle, WA, USA).

\section{RNA extraction and cDNA synthesis}

All the included cell lines were cultured for fewer than ten passages before RNA extraction. Total RNA from LNCaP, NCIH-727, NCIH-720, CNDT2.5, and KRJ1 cells was isolated using a PARIS Kit from Life Technologies, whereas total RNA from the normal fibroblast 1064SK cells was a gift from Prof. M Essand's group (IGP, Rudbeck Laboratory, Uppsala University, Uppsala, Sweden). Furthermore, about 3-5 $\mu$ g total RNA from each frozen specimen was isolated using the RNeasy Mini Kit from Qiagen. The RNA 6000 Nano Kit and the Agilent 2100 Bioanalyzer from Agilent Technologies (Santa Clara, CA, USA) were used to verify the quality and quantity of all isolated total RNAs. The cDNAs was prepared using the cDNA synthesis kit from Bio-Rad for the cell lines and the MuLV reverse transcriptase from Life Technologies for the frozen specimens.

\section{OR51E1 transcript sequencing}

The OR51E1 coding sequence was cloned from the cDNA of NCI-H727 and NCI-H720 cells. PCR was performed using forward primer, 5'-TCA GCT TCT TCA TGA TGG TGG-3' and reverse primer, 5'-CAC TGA CAC CTA GGG CTC TGA-3'. The TOPO TA Cloning Kit from Life Technologies was used for cloning and the OR51E1 coding sequence was analyzed at the Rudbeck Laboratory, Uppsala Genome Centre (Uppsala, Sweden; http://www. genpat.uu.se/facilities/genome_center/). Sequences were then aligned with GenBank published data.

\section{Quantitative real-time PCR}

Gene-specific PCR products were measured in the cell line cDNAs by Agilent Stratagene Mx3005P real-time PCR system with Brilliant II SYBR Green QPCR Master Mix

Published by Bioscientifica Ltd. 
from Agilent Technologies and in the tissue cDNAs by iCycler IQ real-time detection system with iQ SYBR Green Supermix from Bio-Rad. Each PCR contained about $10 \mathrm{ng}$ cDNA when dealing with either cell lines or frozen tumor tissues, in the latter case, by cutting an adequate number of $10 \mu \mathrm{m}$-thick sections comprising at least $75 \%$ tumor cellularity. Relative expression of OR51E1 was evaluated after normalization against $\beta$-actin expression, using the same primers and method as described previously (Leja et al. 2009), where relative fold changes were calculated for each tumor patient in comparison with the median value for normal tissue in all the patients.

\section{Immunohistochemistry and scoring system}

The tissue specimens were fixed in $10 \%$ buffered neutral formalin for $18-20 \mathrm{~h}$ at room temperature, followed by routine processing to paraffin wax. Approximately $4 \mu \mathrm{m}$ thick sections were cut and attached to positively charged glass slides (Superfrost ${ }^{+}$) from Menzel Glässer (Braunschweig, Germany). The sections were microwave treated for $2 \times 5 \mathrm{~min}$ at $700 \mathrm{~W}$ using $50 \mathrm{mM}$ Tris buffer saline, $\mathrm{pH} 8.0$, as retrieval solution and immunostained using a polymer detection system (Dako Cytomation, EnVision + SystemHRP, K4010) from Dako (Glostrup, Denmark). Diaminobenzidine was used as chromogen. The antibodies were diluted in Dako's antibody diluent (S3022). The SSTR antibodies were polyclonal rabbit from Gramsch Laboratories: SSTR2A (SS-800, 1:5000), SSTR3 (SS-850, 1:5000), and SSTR5 (SS-890, 1:5000) and monoclonal rabbit anti-SSTR2A antibody, UMB-1, from Epitomics, Inc. (1:200). OR51E1 polyclonal rabbit antibody (A1854, 1:500) was purchased from LSBio. The sections were incubated with the primary antibodies overnight at room temperature. Meyer's hematoxylin from Histolab (Gothenburg, Sweden) was used for nuclear counterstain. Immunostaining was evaluated, in consecutive immunostained sections, under a light microscope (Axioskop 40) from Carl Zeiss (Oberkochen, Germany) and the percentage of immunoreactive (IR) tumor cells was estimated at a magnification of $\times 400$ using a grid in one of the oculars. At least four randomly selected areas were examined; in small lesions, the entire neoplastic tissue was investigated. Images were obtained at the same magnification using an Axiophot light microscope from Carl Zeiss and AxioVision Rel. 4.5 Software.

Immunohistochemical results for the relevant antibodies were rendered semiquantitatively on a scale from 0 to 3, taking into account the cellular compartmentalization (membrane vs cytoplasm) and the percentage of tumor cells ( $<50$ vs $>50 \%$ ), which slightly modifies a previously released scoring system for SSTR2 (Volante et al. 2007). Briefly, pure cytoplasmic immunoreactivity in $<50 \%$ tumor cells and/or membrane immunoreactivity (partial or circumferential) in $<10 \%$ tumor cells was scored as 0 (S0) (this modification was done in agreement with autoradiography results (Korner et al. 2012)); pure cytoplasmic immunoreactivity in $>50 \%$ tumor cells as 1 (S1); incomplete membranous immunoreactivity $(<50 \%$ of cell circumference) in 10-50\% tumor cells, irrespective of the presence of cytoplasmic immunoreactivity, as 2 (S2); and circumferential membranous immunoreactivity ( $\geq 50 \%$ of cell circumference) in $>50 \%$ tumor cells, irrespective of the presence of cytoplasmic immunoreactivity, as 3 (S3). In addition, we selected the highest immunohistochemical score for SSTR2 from the results obtained using the two different SSTR2 antibodies.

\section{Statistical analysis}

Surgical specimens from 72 primary TCs, 14 primary ACs, and $11 \mathrm{regional} /$ distant metastases were analyzed in all the statistical analyses, whereas another laser-treated primary TC was evaluated for OctreoScan comparison only. In detail, the Wilcoxon-matched pairs signed rank test was used to compare immunohistochemical score for OR51E1 and the highest score among SSTR2, SSTR3, and SSTR5 for each specimen. The Mann-Whitney $U$ test was used to evaluate the statistical difference in OR51E1 mRNA expression between TCs and ACs and to assess the difference in protein expression of each receptor between TCs and ACs, between different stages of tumors, and between smaller $(<3 \mathrm{~cm})$ and larger $(\geq 3 \mathrm{~cm})$ primary LCs, according to the protocol of the American Joint Committee on Cancer classification. The Kruskal-Wallis test, with Dunn's multiple comparison post-hoc analysis, was used to evaluate the difference among SSTR2, SSTR3, and SSTR5 immunohistochemical scores. The Fisher's exact test was used to assess the concordance between the OctreoScan grades and the SSTR2 immunohistochemical scores of the corresponding tumors. The Spearman's correlation analysis was used to access the correlation of immunohistochemical scores analyzed by three different investigators. All the analyses were two-tailed and performed using GraphPad Prism 5 from GraphPad Software (La Jolla, CA, USA). All $P$ values $<0.05$ were considered significant.

\section{Ethics statement}

The entire research protocol was reviewed and approved by the research ethics boards at Uppsala University

Published by Bioscientifica Ltd 
Hospital (Sweden; ref. no. Dnr 2011/051), and the internal revision board of the European Institute of Oncology (Milan, Italy). All the patients included in the study signed a document giving informed consent.

\section{Results}

\section{WT OR51E1 transcript expression in established human LC cell lines}

The sequence analysis showed the absence of any mutation in the OR51E1 coding sequence from both the TC NCI-H727 cells and the AC NCI-H720 cells. The sequence was identical to the original WT OR51E1, which was published on GenBank (accession number: NP_689643.2). Quantitative real-time PCR (QRT-PCR) analysis revealed similar OR51E1 transcript expression between NCI-H727 and NCI-H720 cells. In contrast, no OR51E1 expression was detected in the other investigated established NET cells, i.e. CNDT2.5 and KRJ1 cells (Fig. 1).

\section{OR51E1 transcript expression in both typical and atypical LCs}

QRT-PCR analysis revealed OR51E1 transcript expression in 9 out of 12 TCs and 7 out of 9 ACs $(P=$ NS (not significant) between TCs and ACs), of which 5 out of 12 TCs and 2 out of 9 ACs showed high expression (Table 1).

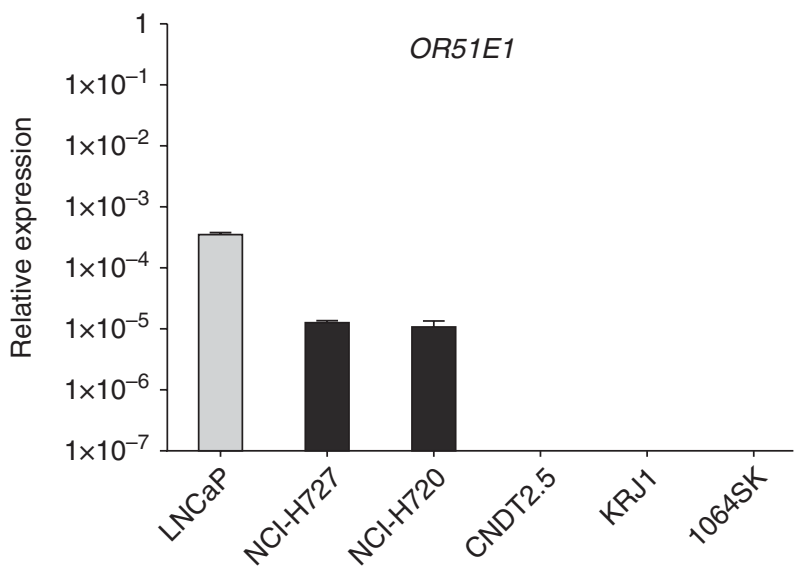

Figure 1

OR51E1 transcript expression in established human neuroendocrine tumor cells. OR51E1 expression was evaluated by QRT-PCR and normalized to $\beta$-actin (set as 1). s.D.s of triplicate samples are shown. $\mathrm{NCl}-\mathrm{H} 727$, typical lung carcinoid; $\mathrm{NCl}-\mathrm{H} 720$, atypical lung carcinoid; KRJ-1 and CNDT2.5, small intestine neuroendocrine carcinomas. LNCaP cells, from a prostate carcinoma, were used as a positive control and 1064 SK cells, from normal skin fibroblast, were used as a negative control.
Table 1 Relative OR51E1 transcript expression in different types of lung cancer cells

\begin{tabular}{|c|c|c|c|c|}
\hline & - & + & ++ & +++ \\
\hline $\operatorname{TCs}(n=12)$ & $3 / 12$ & $3 / 12$ & $1 / 12$ & $5 / 12$ \\
\hline $\operatorname{ACs}(n=9)$ & $2 / 9$ & $3 / 9$ & $2 / 9$ & $2 / 9$ \\
\hline $\operatorname{SCLCs}(n=10)$ & $3 / 10$ & $6 / 10$ & $1 / 10$ & $0 / 10$ \\
\hline $\operatorname{ADCs}(n=5)$ & $3 / 5$ & $2 / 5$ & $0 / 5$ & $0 / 5$ \\
\hline $\operatorname{SCCs}(n=5)$ & $1 / 5$ & $3 / 5$ & $1 / 5$ & $0 / 5$ \\
\hline
\end{tabular}

TC, typical carcinoid; AC, atypical carcinoid; SCLC, small-cell lung carcinoma; $A D C$, adenocarcinoma; SCC, squamous cell carcinoma; - , negative expression; + , low expression; ++ , intermediate expression; +++ , high expression

Predominant OR51E1 expression was detected in both the TC and AC tumor cells compared with the normal surrounding bronchopulmonary cells (Table 2). Furthermore, OR51E1 transcript expression was detected in 7/10 SCLCs, 2/5 lung adenocarcinomas, and 4/5 lung squamous cell carcinomas, whereas high OR51E1 expression was not observed in these carcinomas (Table 1).

\section{Immunohistochemistry and statistical analysis}

Lung carcinoids Based on our proposed scoring system (SO-S3), OR51E1 and SSTR2, SSTR3, and SSTR5 expressions on all the LC tumor sections were evaluated by three different investigators ( $\mathrm{T} C, \mathrm{~L} \mathrm{G}$, and $\mathrm{A} \mathrm{V} \mathrm{T}$ ). The immunohistochemical scores for each of the four receptors showed high correlation (Spearman's correlation coefficient $\rho>0.9$ ) between any two different investigators of the three. Thus, this scoring system is highly reproducible for the present investigation. Furthermore, we used the median scores obtained by the three investigators, for each receptor and on each tumor specimen, during our subsequent statistical analyses.

Table 2 Relative OR51E1 transcript expression in TC and AC cells in comparison with normal bronchopulmonary cells from the same patients

\begin{tabular}{lcccc}
\hline TCs $(n=4)$ & T1 & +++ & N1 & - \\
& T2 & +++ & N2 & - \\
& T3 & +++ & N3 & - \\
ACs $(n=4)$ & T4 & - & N4 & + \\
& T1 & +++ & N1 & - \\
& T2 & + & N2 & - \\
& T3 & - & N3 & - \\
& T4 & - & N4 & -
\end{tabular}

TC, typical carcinoid; AC, atypical carcinoid; T, carcinoid tumor cells; N, normal bronchopulmonary cells; - negative expression; + , low expression; ++ , intermediate expression; +++ , high expression.

Published by Bioscientifica Ltd. 


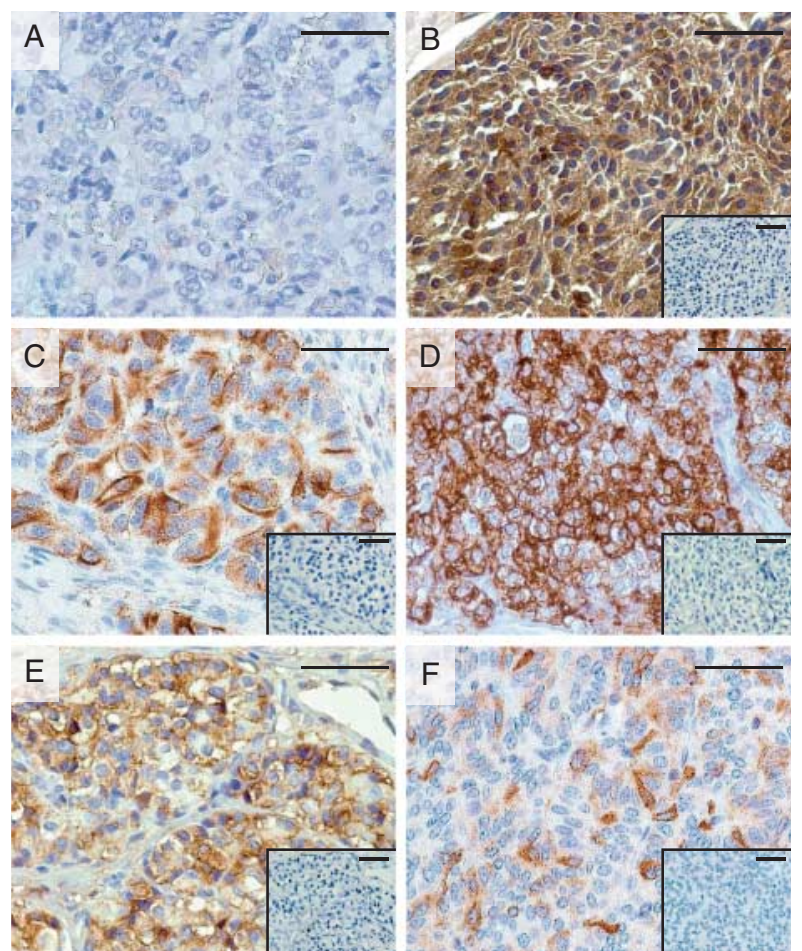

Figure 2

OR51E1 immunostaining in lung carcinoids. Immunostaining patterns in the neoplastic cells of typical carcinoids are illustrated as non-immunoreactive, score 0 (A); pure cytoplasmic, score 1 (B); incomplete membranous in $<50 \%$ of tumor cells, score 2 (C); and circumferential membranous in $>50 \%$ of tumor cells, score 3 (D). Representative immunostaining in lymph node metastasis, score 3, from a typical carcinoid (E), and in liver metastasis, score 2, from an atypical carcinoid (F), are also shown. Neutralized immunostaining in the same cell clusters is shown in each inset. Bar $=50 \mu \mathrm{m}$

OR51E1 immunostaining according to the scoring system is depicted in Fig. 2. OR51E1 protein was highly expressed mainly in the tumor cell membrane of both primary TCs and ACs and of the regional/distant metastases, with an inverse correlation being detected between OR51E1 and SSTRs. Indeed, the SO group included most SSTR-negative tumors relative to OR51E1 cases, whereas the S3 group harbored the highest distribution of OR51E1positive tumors relative to SSTR cases, with intermediate distributions being found in S1 and S2 groups (Fig. 3). In total, OR51E1, SSTR2, SSTR3, and SSTR5 were detected (corresponding to immunohistochemical scores S1, S2, and S3) in $85,71,25$, and $39 \%$ of primary TCs and in 86 , 79,43 , and $36 \%$ of primary ACs respectively whereas $79 \%$ (57/72) of primary TCs had higher or equal scores for OR51E1 compared with highest score for SSTR2, SSTR3, and SSTR5 $(P=0.0083)$ (Table 3). This percentage for the
TC lymph node metastases was 70\% (7/10). Although not statistically significant $(P=0.1246)$, this percentage for the primary ACs was $86 \%(12 / 14)$, whereas the AC liver metastasis was categorized as S2 for OR51E1 and S3 for SSTR2. Furthermore, no significant difference in OR51E1 scores, or in each SSTR score, was detected between primary TCs and ACs, between different stages of disease, i.e. primaries and metastases, or between smaller $(<3 \mathrm{~cm})$ and larger $(\geq 3 \mathrm{~cm})$ primary LC tumors. Thus, OR51E1 and each SSTR were distributed uniformly across the entire spectrum of LCs.
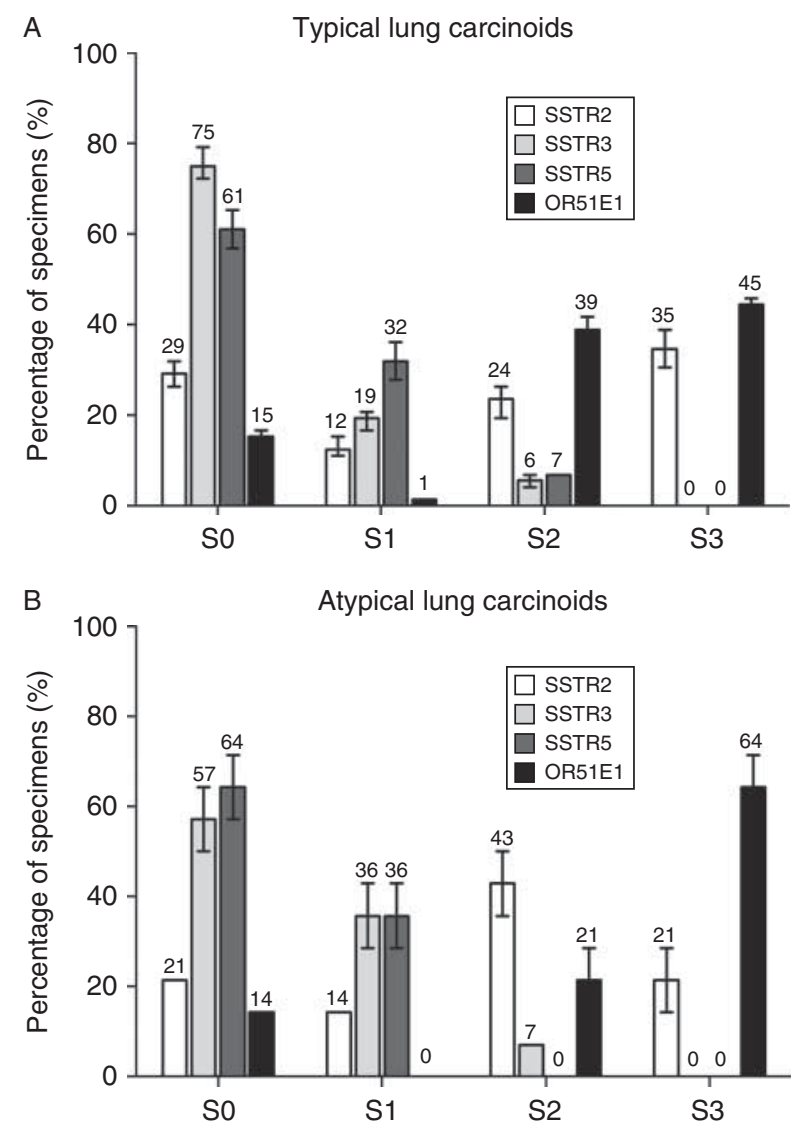

Figure 3

Summary of SSTR2, SSTR3, SSTR5, and OR51E1 immunohistochemical scores in primary typical (A) and atypical carcinoids (B). $X$-axis shows score 0 (SO) to S3, whereas $y$-axis shows the percentage of analyzed tumors (indicated above the columns), which were immunostained for each receptor and classified in each scoring category. The number of tumors that correlates with each percentage, in 72 primary typical carcinoids, is S0: $21,54,44,11$ vs S1: 9, 14, 23, 1 vs S2: 17, 4, 5, 28 vs S3: 25, 0, 0, 32 for SSTR2, SSTR3, SSTR5, and OR51E1 respectively and, in 14 primary atypical carcinoids, is S0: 3, 8, 9, 2 vs S1: 2, 5, 5, 0 vs S2: 6, 1, 0, 3 vs S3: 3, 0, 0, 9 for SSTR2, SSTR3, SSTR5, and OR51E1 respectively. All the numerical values as described above represent calculations based on the median immunohistochemical scores obtained by the three investigators, whereas the ranges of the scores are indicated by the error bars for each column.

Published by Bioscientifica Ltd. 
Table 3 Comparison of OR51E1 immunohistochemical score with the highest SSTR2, SSTR3, and SSTR5 immunohistochemical score in lung carcinoids

\begin{tabular}{lll}
\hline Type & Primary or metastasis \\
${$\cline { 1 - 1 }$} }$ & Primary \\
TC & Lymph node metastasis \\
AC & Primary \\
AC & Liver metastasis \\
\hline
\end{tabular}

\begin{tabular}{c}
\hline OR51E1 $>$ SSTRs \\
\hline $27 / 72(37 \%)$ \\
$4 / 10(40 \%)$ \\
$8 / 14(57 \%)$ \\
$0 / 1$ \\
\hline
\end{tabular}

\begin{tabular}{c}
\hline OR51E1 $=$ SSTRS \\
\hline $30 / 72(42 \%)$ \\
$3 / 10(30 \%)$ \\
$4 / 14(29 \%)$ \\
$1 / 1$ \\
\hline
\end{tabular}

\begin{tabular}{c}
\hline OR51E1 $<$ SSTRs \\
\hline $15 / 72(21 \%)$ \\
$3 / 10(30 \%)$ \\
$2 / 14(14 \%)$ \\
$0 / 1$ \\
\hline
\end{tabular}

\begin{tabular}{l}
\hline $\boldsymbol{P}$ value \\
\hline 0.0083 \\
NS \\
NS \\
NA
\end{tabular}

TC, typical carcinoid; AC, atypical carcinoid; NS, not significant; NA, not applicable.

Moreover, the analysis of 22 SSTR-non-IR (S0) lesions revealed that membrane localization (S2 or S3) of OR51E1 was detected in the tumor cells in 10 out of 17 primary TCs, 2 out of 3 TC lymph node metastases, and 1 out of 2 primary ACs. Furthermore, 7 out of 9 primary TCs, 2 out of 3 TC lymph node metastases, and 2 out of 2 primary ACs showed membrane OR51E1 immunohistochemical pattern (S2 or S3), where SSTR2 was predominantly localized in the cytoplasm (S1) (Fig. 4).

In addition, we observed higher SSTR2 expression compared with SSTR3 and SSTR5 $(P<0.05)$ in both types of LCs, whereas no difference was detected between SSTR3 and SSTR5. Between the immunohistochemical scores obtained using the two SSTR2 antibodies, 16 out of 72 primary TCs and 5 out of 14 primary ACs showed higher scores when the clone UMB-1 SSTR2 antibody from Epitomics, Inc. was used, whereas 8 out of 72 TCs and 2 out of 14 ACs showed higher scores using the SSTR2 antibody from Gramsch Laboratories.

Controls Distinct cells with membrane immunoreactivity were identified in the pancreatic islets and in prostate carcinoma cells for SSTRs and OR51E1 respectively. No immunoreactivity was seen after replacing the relevant primary antibodies with the non-related rabbit immunoglobulin or after the neutralization test.

\section{OR51E1 protein is expressed in LCs with negative OctreoScan}

A comparison between functional imaging and immunohistochemical results was performed on 12 primary TCs, two primary ACs, and five metastases, i.e. four lymph node metastases from TCs and one liver metastasis from an AC, for which OctreoScan data were available. In total, 4 out of 6 OctreoScan-negative lesions were immunohistochemically scored 0 (S0) for SSTR2, 4 out of 6 OctreoScan grade 1 lesions were scored 1 (S1), and 7 out of 7 OctreoScan grades 2-4 lesions were scored 2-3 (S2-S3). Thus, the concordance rate was 79\% (15 out of 19 lesions) between the OctreoScan grades and the corresponding SSTR2 immunohistochemical scores $(P<0.001)$.

At variance with SSTRs, OR51E1 immunoreactivity was uniformly distributed across the tumors for which OctreoScan data were available regardless of uptake levels (Table 4). Indeed, OR51E1 scores (S2-S3) were higher than SSTR2, SSTR3, and SSTR5 (SO-S1) in 5 out of 6 OctreoScannegative TC lesions (five primary tumors and one lymph node metastasis). OR51E1 showed high scores (S2-S3) in 5 out of 6 OctreoScan grade 1 lesions and in 7 out of 7 OctreoScan grade 2-4 lesions, where moreover, 3 out of 6 grade 1 lesions and 2 out of 7 grade $2-4$ lesions presented higher scores for OR51E1 than all the SSTR subtypes $(P=0.042$ for OR51E1 scores vs highest SSTR scores, in all the 19 LC lesions).

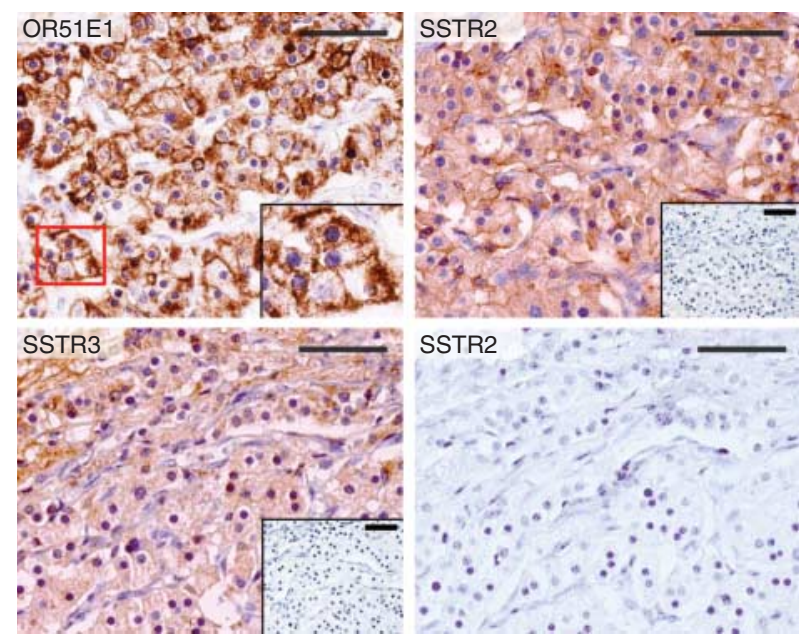

Figure 4

Representative immunostaining with higher OR51E1 score compared with SSTR2, SSTR3, and SSTR5 in a primary typical carcinoid. OR51E1 shows membrane pattern staining in the tumor cells (score 3 ). The inset highlights OR51E1 membranous staining with a higher magnification $(\times 800)$, of the tumor cells marked by the red frame. SSTR2 (photo obtained from immunostaining by using anti-SSTR2, clone UMB1) and SSTR3 exhibit mainly cytoplasmic staining in these tumor cells (score 1), for which neutralized immunostaining are shown in the insets. The tumor is non-immunoreactive for SSTR5 (score 0). Bar $=50 \mu \mathrm{m}$. 
Table 4 SSTR2, SSTR3, SSTR5, and OR51E1 immunohistochemical scores in lung carcinoids with OctreoScan data

\begin{tabular}{|c|c|c|c|c|c|c|c|}
\hline Patient & Type & Primary or metastasis & OctreoScan & SSTR2 & SSTR3 & SSTR5 & OR51E1 \\
\hline 1 & TC & Primary & No uptake & so & so & so & S3 \\
\hline 2 & TC & Primary & No uptake & So & So & So & S2 \\
\hline 3 & $\mathrm{TC}$ & Primary & No uptake & SO & So & So & so \\
\hline \multirow[t]{2}{*}{4} & $\mathrm{TC}$ & Primary & No uptake & S1 & So & So & S2 \\
\hline & $\mathrm{TC}$ & Primary & No uptake & S1 & S1 & So & S3 \\
\hline \multirow[t]{2}{*}{5} & $\mathrm{TC}$ & Lymph node metastasis & No uptake & SO & so & So & S2 \\
\hline & $\mathrm{TC}$ & Lymph node metastasis & Grade 1 & S1 & S2 & S2 & S2 \\
\hline 6 & $\mathrm{TC}$ & Primary & Grade 1 & S2 & so & so & so \\
\hline \multirow[t]{2}{*}{7} & TC & Primary & Grade 1 & S2 & so & S1 & S2 \\
\hline & $\mathrm{TC}$ & Lymph node metastasis & Grade 1 & S1 & S1 & S1 & S3 \\
\hline 8 & TC & Primary & Grade 1 & S1 & So & so & S2 \\
\hline 9 & $\mathrm{TC}$ & Primary & Grade 1 & S1 & So & So & S3 \\
\hline 10 & TC & Primary & Grade 3 & S3 & S1 & S1 & S3 \\
\hline \multirow[t]{2}{*}{11} & $\mathrm{TC}$ & Primary & Grade 3 & S2 & so & S1 & S3 \\
\hline & $\mathrm{TC}$ & Lymph node metastasis & Grade 3 & S2 & so & S1 & S3 \\
\hline 12 & $\mathrm{TC}$ & Primary & Grade 4 & S2 & so & so & S2 \\
\hline \multirow[t]{2}{*}{13} & $A C$ & Primary & Grade 2 & S3 & so & So & S2 \\
\hline & $A C$ & Liver metastasis & Grade 3 & S3 & so & S1 & S2 \\
\hline 14 & $A C$ & Primary & Grade 3 & S3 & S1 & S1 & S3 \\
\hline
\end{tabular}

TC, typical carcinoid; AC, atypical carcinoid; S0, score 0 , non-immunoreactive specimens, pure cytoplasmic immunoreactivity in $<50 \%$ of tumor cells and membrane immunoreactivity (partial or circumferential) in $<10 \%$ of tumor cells; S1, score 1 , pure cytoplasmic immunoreactivity in $>50 \%$ of tumor cells; S2, score 2, incomplete membranous immunoreactivity ( $<50 \%$ of cell circumference) in $10-50 \%$ of tumor cells, irrespective of the presence of cytoplasmic immunoreactivity; S3, score 3, circumferential membranous immunoreactivity ( $\geq 50 \%$ of cell circumference) in $>50 \%$ of tumor cells, irrespective of the presence of cytoplasmic immunoreactivity. Octreoscan data were graded according to the method described by Kwekkeboom et al. 2005 .

\section{Discussion}

SSTRs may be variably expressed in typical and atypical LCs (Papotti et al. 2001, Pelosi et al. 2006, Righi et al. 2010, Tsuta et al. 2012) and may be used in both their diagnosis and treatment. However, novel molecular targets are needed to improve the clinical management of these patients. Here, we indicated that OR51E1, a G-protein coupled receptor, showed a higher or similar expression compared with SSTR2, SSTR3, and SSTR5 in 79\% (57 out of 72) of primary TCs, $86 \%$ (12 out of 14 ) of primary ACs, and in $73 \%$ (8 out of 11) LC metastases. High membrane OR51E1 expression was found in 10 out of 17 primary TCs and 1 out of 2 primary ACs that were non-IR for SSTRs and moreover in 5 out of 6 OctreoScan-negative TC lesions. Furthermore, OR51E1 showed more extensive membrane localization than SSTRs in 7 out of 9 primary TCs and 2 out of 2 primary ACs exhibiting cytoplasmic immunostaining for SSTRs and moreover in 3 out of 6 OctreoScan grade 1 TC lesions. These findings indicate that OR51E1 may play an important role as a potential novel target in the diagnosis of both TCs and ACs, irrespective of their SSTR expression.

The predominant OR51E1 expression by both immunohistochemistry and QRT-PCR in tumors when compared with normal lung tissue, its distribution across the spectrum of LCs, and the lack of mutations in the relevant gene supports the potential of OR51E1 as a stable molecule in these tumors to target for diagnostic purposes. For the OR51E1 receptor, the relevance of membrane localization to diagnosis of NETs is heralded by the use of other G-protein-coupled receptors, e.g. SSTRs, as mainly membrane rather than cytoplasmic immunoreactivity should be considered for a standardized and reliable clinical report (Volante et al. 2007). In our study, OR51E1 has shown more extensive membrane localization compared with SSTR2, SSTR3, and SSTR5, which are targeted by most of the clinically available SSAs. These findings indicated that OR51E1 might display a higher uptake in LC cells, compared with the SSA-based diagnostics, after developing appropriate procedures of immuno-positron emission tomography (PET; Knowles \& Wu 2012) by means of OR51E1 monoclonal antibodies with high receptor binding affinity. Indeed, OR51E1 has shown an advantage over SSTRs as it has prevalence across the entire spectrum of LCs, even in OctreoScan-negative and/or SSTR non-IR LCs, independent of tumor size. This heightens the opportunity to use OR51E1 as a complement to SSTRs in diagnosing these tumors.

In conclusion, our study indicates that OR51E1 may be a potential novel target for LCs, especially when dealing with SSTR-negative tumor patients. Tumor diagnosis

Published by Bioscientifica Ltd. 
based on OR51E1 immuno-PET analysis is an interesting possibility for better management of these challenging tumor patients irrespective of their SSTR status, although further studies are required to test these possibilities.

\section{Declaration of interest}

The authors declare that there is no conflict of interest that could be perceived as prejudicing the impartiality of the research reported.

\section{Funding}

This work was supported by funding from Erik, Karin och Gösta Selander Stiftelse (2010 and 2012). The funders had no role in study design, data collection, and analysis; decision to publish; or preparation of the manuscript.

\section{Author contribution statement}

V G, T C, G P, and A V T conceived and designed the experiments. T C, $L G, G P$, and $A \vee T$ performed the experiments. V G, T C, L G, G P, and A $\vee T$ analyzed the data. $\vee G, K$ O, G $P$, and $A \vee T$ contributed reagents/materials and analysis tools. V G, T C, L G, G P, and A V T wrote the paper.

\section{Acknowledgements}

The authors thank Su-Chen Li, Department of Medical Sciences, Endocrine Oncology, Uppsala University (Uppsala, Sweden) for critical reading of the manuscript. They are also grateful for the financial support from the above-mentioned funders.

Distinction: This original project was presented orally at the 10th Annual European Neuroendocrine Tumor Society meeting in Barcelona, Spain, 6-8 March 2013 and was awarded the first prize.

\section{References}

Ache BW \& Young JM 2005 Olfaction: diverse species, conserved principles. Neuron 48 417-430. (doi:10.1016/j.neuron.2005.10.022)

Beasley MB, Thunnissen FB, Hasleton PS, Barbareschi M, Pugatch B, Geisinger K, Brambilla E, Gazdar A \& Travis WD 2004 Carcinoid tumour. In World Health Organization Classification of Tumours. Pathology and Genetics of Tumours of the Lung, Pleura, Thymus and Heart, 3rd edn, pp 59-62. Eds WD Travis, E Brambilla, HK Muller-Hermelink \& CC Harris. Lyon, France: IARC Press.

van Buren G II, Rashid A, Yang AD, Abdalla EK, Gray MJ, Liu W, Somcio R, Fan F, Camp ER, Yao JC et al. 2007 The development and characterization of a human midgut carcinoid cell line. Clinical Cancer Research 13 4704-4712. (doi:10.1158/1078-0432.CCR-06-2723)

Cui T, Tsolakis AV, Li SC, Cunningham JL, Lind T, Oberg K \& Giandomenico V 2013 Olfactory receptor 51E1 protein as a potential novel tissue biomarker for small intestine neuroendocrine carcinomas. European Journal of Endocrinology 168 253-261. (doi:10.1530/EJE12-0814)

van Essen M, Krenning EP, Bakker WH, de Herder WW, van Aken MO \& Kwekkeboom DJ 2007a Peptide receptor radionuclide therapy with 177Lu-octreotate in patients with foregut carcinoid tumours of bronchial, gastric and thymic origin. European Journal of Nuclear Medicine and Molecular Imaging 34 1219-1227. (doi:10.1007/s00259. 006-0355-4) van Essen M, Krenning EP, De Jong M, Valkema R \& Kwekkeboom DJ 2007b Peptide receptor radionuclide therapy with radiolabelled somatostatin analogues in patients with somatostatin receptor positive tumours. Acta Oncologica 46 723-734. (doi:10.1080/02841860701441848)

Filosso PL, Rena O, Donati G, Casadio C, Ruffini E, Papalia E, Oliaro A \& Maggi G 2002a Bronchial carcinoid tumors: surgical management and long-term outcome. Journal of Thoracic and Cardiovascular Surgery 123 303-309. (doi:10.1067/mtc.2002.119886)

Filosso PL, Ruffini E, Oliaro A, Papalia E, Donati G \& Rena O $2002 b$ Long-term survival of atypical bronchial carcinoids with liver metastases, treated with octreotide. European Journal of Cardio-Thoracic Surgery 21 913-917. (doi:10.1016/S1010-7940(02)00055-6)

Fuessel S, Weigle B, Schmidt U, Baretton G, Koch R, Bachmann M, Rieber EP, Wirth MP \& Meye A 2006 Transcript quantification of Dresden G protein-coupled receptor (D-GPCR) in primary prostate cancer tissue pairs. Cancer Letters 236 95-104. (doi:10.1016/j.canlet.2005.05.005)

Fujita Y, Takahashi T, Suzuki A, Kawashima K, Nara F \& Koishi R 2007 Deorphanization of Dresden $G$ protein-coupled receptor for an odorant receptor. Journal of Receptor and Signal Transduction Research 27 323-334. (doi:10.1080/10799890701534180)

Gustafsson BI, Kidd M, Chan A, Malfertheiner MV \& Modlin IM 2008 Bronchopulmonary neuroendocrine tumors. Cancer 113 5-21. (doi:10.1002/cncr.23542)

Knowles SM \& Wu AM 2012 Advances in immuno-positron emission tomography: antibodies for molecular imaging in oncology. Journal of Clinical Oncology 30 3884-3892. (doi:10.1200/JCO.2012.42.4887)

Korner M, Waser B, Schonbrunn A, Perren A \& Reubi JC 2012 Somatostatin receptor subtype $2 \mathrm{~A}$ immunohistochemistry using a new monoclonal antibody selects tumors suitable for in vivo somatostatin receptor targeting. American Journal of Surgical Pathology 36 242-252. (doi:10.1097/PAS.0b013e31823d07f3)

Kwekkeboom DJ, Teunissen JJ, Bakker WH, Kooij PP, de Herder WW, Feelders RA, van Eijck CH, Esser JP, Kam BL \& Krenning EP 2005 Radiolabeled somatostatin analog [177Lu-DOTA0,Tyr3)octreotate in patients with endocrine gastroenteropancreatic tumors. Journal of Clinical Oncology 23 2754-2762. (doi:10.1200/JCO.2005.08.066)

Kwekkeboom DJ, de Herder WW \& Krenning EP 2011 Somatostatin receptor-targeted radionuclide therapy in patients with gastroenteropancreatic neuroendocrine tumors. Endocrinology and Metabolism Clinics of North America 40 173-185, ix. (doi:10.1016/j.ecl.2010.12.003)

Leja J, Essaghir A, Essand M, Wester K, Oberg K, Tötterman TH, Lloyd R, Vasmatzis G, Demoulin JB \& Giandomenico V 2009 Novel markers for enterochromaffin cells and gastrointestinal neuroendocrine carcinomas. Modern Pathology 22 261-272. (doi:10.1038/modpathol.2008.174)

Modlin IM, Kidd M, Pfragner R, Eick GN \& Champaneria MC 2006 The functional characterization of normal and neoplastic human enterochromaffin cells. Journal of Clinical Endocrinology and Metabolism 91 2340-2348. (doi:10.1210/jc.2006-0110)

Olender T, Lancet D \& Nebert DW 2008 Update on the olfactory receptor (OR) gene superfamily. Human Genomics 3 87-97. (doi:10.1186/ 1479-7364-3-1-87)

Papotti M, Croce S, Bello M, Bongiovanni M, Allia E, Schindler M \& Bussolati G 2001 Expression of somatostatin receptor types 2, 3 and 5 in biopsies and surgical specimens of human lung tumours. Correlation with preoperative octreotide scintigraphy. Virchows Archiv: an International Journal of Pathology 439 787-797. (doi:10.1007/ s004280100494)

Pelosi G, Volante M, Papotti M, Sonzogni A, Masullo M \& Viale G 2006 Peptide receptors in neuroendocrine tumors of the lung as potential tools for radionuclide diagnosis and therapy. Quarterly Journal of Nuclear Medicine and Molecular Imaging 50 272-287.

Righi L, Volante M, Tavaglione V, Bille A, Daniele L, Angusti T, Inzani F, Pelosi G, Rindi G \& Papotti M 2010 Somatostatin receptor tissue distribution in lung neuroendocrine tumours: a clinicopathologic and immunohistochemical study of 218 'clinically aggressive' cases. Annals of Oncology 21 548-555. (doi:10.1093/annonc/mdp334) 
Saito H, Chi Q, Zhuang H, Matsunami H \& Mainland JD 2009 Odor coding by a mammalian receptor repertoire. Science Signaling 2 ra9. (doi:10.1126/scisignal.2000016)

Tsuta K, Wistuba II \& Moran CA 2012 Differential expression of somatostatin receptors 1-5 in neuroendocrine carcinoma of the lung. Pathology, Research and Practice 208 470-474. (doi:10.1016/j.prp.2012. 05.014)

Vanti WB, Nguyen T, Cheng R, Lynch KR, George SR \& O'Dowd BF 2003 Novel human G-protein-coupled receptors. Biochemical and Biophysical Research Communications 305 67-71. (doi:10.1016/S0006-291X (03)00709-5)

Volante M, Brizzi MP, Faggiano A, La Rosa S, Rapa I, Ferrero A, Mansueto G, Righi L, Garancini S, Capella C et al. 2007 Somatostatin receptor type $2 \mathrm{~A}$ immunohistochemistry in neuroendocrine tumors: a proposal of scoring system correlated with somatostatin receptor scintigraphy. Modern Pathology 20 1172-1182. (doi:10.1038/modpathol.3800954)

Wang J, Weng J, Cai Y, Penland R, Liu M \& Ittmann M 2006 The prostatespecific G-protein coupled receptors PSGR and PSGR2 are prostate cancer biomarkers that are complementary to $\alpha$-methylacyl-CoA racemase. Prostate 66 847-857. (doi:10.1002/pros.20389)

Weigle B, Fuessel S, Ebner R, Temme A, Schmitz M, Schwind S, Kiessling A, Rieger MA, Meye A, Bachmann M et al. 2004 D-GPCR: a novel putative $\mathrm{G}$ protein-coupled receptor overexpressed in prostate cancer and prostate. Biochemical and Biophysical Research Communications $\mathbf{3 2 2}$ 239-249. (doi:10.1016/j.bbrc.2004.07.106)

Weng J, Wang J, Hu X, Wang F, Ittmann M \& Liu M 2006 PSGR2, a novel G-protein coupled receptor, is overexpressed in human prostate cancer. International Journal of Cancer 118 1471-1480. (doi:10.1002/ijc.21527)

Received in final form 13 August 2013

Accepted 22 August 2013

Accepted Preprint published online 22 August 2013
C 2013 Society for Endocrinology Printed in Great Britain
Published by Bioscientifica Ltd. 